\title{
Transient stability of large helical device conductor with and without aluminum stabilizer (2) - Numerical results
}

\section{AUTHOR(S):}

Shirai, Y; Ohya, M; Ikuta, R; Shiotsu, M; Imagawa, S

\section{CITATION:}

Shirai, Y ... [et al]. Transient stability of large helical device conductor with and without aluminum stabilizer (2) - Numerical results. IEEE TRANSACTIONS ON APPLIED

SUPERCONDUCTIVITY 2007, 17(2): 2458-2461

\section{ISSUE DATE:}

2007-06

URL:

http://hdl.handle.net/2433/50271

\section{RIGHT:}

(c)2007 IEEE. Personal use of this material is permitted. However, permission to reprint/republish this material for advertising or promotional purposes or for creating new collective works for resale or redistribution to servers or lists, or to reuse any copyrighted component of this work in other works must be obtained from the IEEE. 


\title{
Transient Stability of Large Helical Device Conductor With and Without Aluminum Stabilizer (2)—Numerical Results
}

\author{
Y. Shirai, Member, IEEE, M. Ohya, R. Ikuta, M. Shiotsu, and S. Imagawa, Member, IEEE
}

\begin{abstract}
Numerical simulations were carried out on the transient stability of large-scale composite superconductors against a thermal disturbance, that is, a LHD conductor, which consists of a $\mathrm{NbTi} / \mathrm{Cu}$ Rutherford cable, a pure aluminum stabilizer, and a copper sheath around the composite. The simulations were also performed on an Al-less test conductor, which is a LHD conductor without the Al stabilizer and a half of the copper sheath. The recovery and propagation characteristics of an initiated normal zone were simulated to know the effect of the Al stabilizer on the transient stability of the LHD conductor cooled by Liq.He II. The normal zone propagation initiating current at a certain magnetic flux density for the LHD conductor was compared with that for the Al-less test conductor. Asymmetrical propagation of the normal zone appears even in the LHD based conductor without the Al stabilizer. The range of the transport current, which lead to the one-side propagation, is narrower than those for the LHD conductor. It is confirmed that the Al stabilizer in LHD conductor plays main role in the asymmetrical normal zone propagation. The high performance of the He II cooling in the transient state for the Al-less test conductor makes the normal zone initiating current up to the same level of that for the super-stabilized LHD conductor. It is confirmed that only a slight area of the thick aluminum works as a stabilizer at the transient state because of its low magnetic diffusion factor.
\end{abstract}

Index Terms-Aluminum stabilized superconductor, hall effect, superfluid helium, transient stability.

\section{INTRODUCTION}

$\mathbf{L}$ ARGE HELICAL DEVICE (LHD) is a Heliotron-type fusion experimental one, whose helical coil conductor was designed as a super-stabilized superconducting conductor consists of a NbTi/Cu Rutherford cable, a pure aluminum stabilizer, and a copper sheath with chemically oxidized surface. It has an asymmetric configuration in cross-sectional area as shown in Fig. 1. While the excitation tests of the LHD cooled by Liq. He I, the "dynamic one-side propagation" of a traveling normal zone initiated by a certain thermal disturbance was observed at a current and a magnetic field slightly lower than the designed operating point [1]. This "traveling normal zone" did not lead to the quench of the coil. However, in order to achieve a higher

Manuscript received August 29, 2006. This work was supported in part by the LHD Joint Research Project of the National Institute for Fusion Science (NIFS), Japan, and in part by the 21st COE Program of Kyoto University.

Y. Shirai, M. Ohya, R. Ikuta, and M. Shiotsu are with the Department of Energy Science and Technology, Kyoto University, Gokasho, Uji, Kyoto 6110011, Japan (e-mail: shirai@energy.kyoto-u.ac.jp).

S. Imagawa is with the National Institute for Fusion Science, Toki, Gifu 5095292, Japan (e-mail: Imagawa@LHD.nifs.ac.jp).

Digital Object Identifier 10.1109/TASC.2007.898527

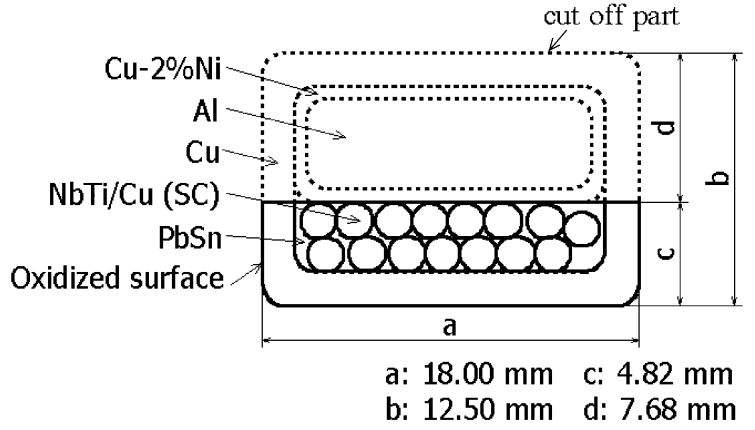

Fig. 1. Cross-sectional view of the test conductor which is cut off the aluminum stabilizer part from LHD conductor.

magnetic field, sub-cooled He I cooling is planned and prepared now. Superfluid liquid helium (He II) cooling for the LHD would be one of the alternatives to get a higher magnetic field. It is necessary to clear the problems on the LHD coil cooled by sub-cooled He I and He II.

On this viewpoint, the stability tests of the small LHD conductor test coil immersed in pressurized He II and sub-cooled He I were carried out and reported [2], [3]. The advantage of the He II cooling became clear compared with the He I cooling. The dynamic one-side propagation appeared in wider current range with the He II cooling.

The traveling normal zone appeared in the aluminum-stabilized conductor has been numerically studied by, for example, Devred [4], Kupferman [5], and Lee [6]. However, the asymmetrical propagation of "traveling normal zone" is peculiar to the LHD conductor. Yanagi [7] has suggested that the Hall electric field generated in the aluminum stabilizer can explain the asymmetrical propagation of the normal zone. The simulation code for the stability of the LHD conductor has been developed by Noguchi [8]. However, the mechanism of the dynamic one-side propagation has not been cleared yet.

The authors have also developed the numerical code to predict the dynamic one-side propagation of LHD conductor cooled by saturated, sub-cooled and superfluid helium [9]. The results showed the asymmetrical propagation of a short finite length normal zone due to the Hall current.

On the other hand, in order to clear the effects of the aluminum stabilizer on the transient stability, the stability test was carried out using the LHD conductor whose aluminum stabilizer is cut off [10]. In this paper, the simulation studies on the transient stability of the Al-less test conductor are described and the effect of the aluminum stabilizer of the LHD conductor is discussed. 


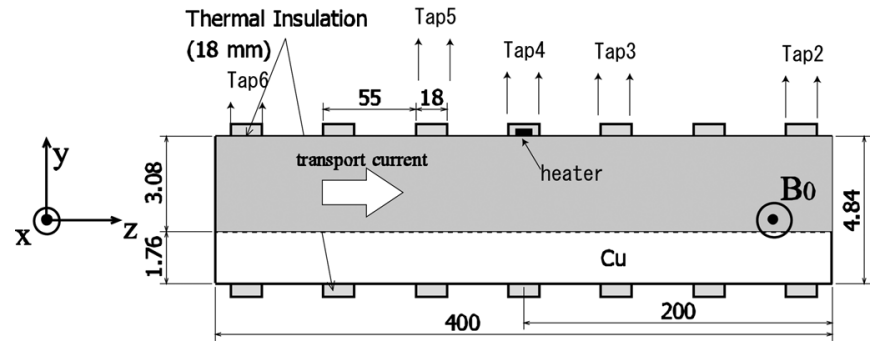

Fig. 2. Simulation model of the test conductor corresponding to the experiment. Thermal insulation simulates the spacers of the LHD conductor. A heater is set at the center of test part of Rutherford side.

\section{Simulation Model}

The cross-sectional view of the LHD conductor is shown in Fig. 1. The LHD conductor is a composite superconductor, which consists of a NbTi/Cu Rutherford cable, a pure aluminum stabilizer and a copper sheath. The conductor has rectangular shape with $18.0 \mathrm{~mm} \times 12.5 \mathrm{~mm}$ size and a $\mathrm{Cu}$-oxide layer covers the outer surface of it. $\mathrm{Cu}-2 \% \mathrm{Ni}$ is used as the clad material around the aluminum stabilizer in order to reduce a transverse Hall current and improve the cryogenic stability.

The numerical analysis was performed on the Al-less test conductor as shown in Fig. 1 to clear the effects of the aluminum stabilizer on the transient stability.

Fig. 2 shows the two-dimensional simulation model based on the test conductor and the axis orientation. The transport current $\boldsymbol{I}$ flows along the z-axis, and the external magnetic field $\boldsymbol{B}_{0}$ is applied in the positive x-direction. The conductor surface is thermally insulated by Kapton tape in parts of $18 \mathrm{~mm}$ with $5 \mathrm{~mm}$ interval, then the exposure ratio of the conductor surface to the coolant is $67 \%$ corresponding to the spacers of LHD helical coils. Voltage taps and a heater (under the insulation tapes at tap4) are set corresponding to the experiment.

The basic electro-magnetic equations of the conductor are given as

$$
\begin{aligned}
\mu_{0} \frac{\partial \boldsymbol{J}}{\partial \boldsymbol{t}} & =\nabla^{2} \boldsymbol{E}_{1}-\nabla\left(\nabla \cdot \boldsymbol{E}_{1}\right) \\
\mu_{0} \frac{\partial \boldsymbol{J}_{h}}{\partial \boldsymbol{t}} & =\nabla^{2} \boldsymbol{E}_{h}-\nabla\left(\nabla \cdot \boldsymbol{E}_{h}\right) \\
\boldsymbol{E}_{1} & =\rho \boldsymbol{J} \\
\boldsymbol{E}_{h} & =\rho \boldsymbol{J}_{h}-\boldsymbol{R}_{H}\left(\boldsymbol{J}+\boldsymbol{J}_{h}\right) \times \boldsymbol{B}_{0}
\end{aligned}
$$

where $\boldsymbol{J}$ and $\boldsymbol{J}_{h}$ denote the transport and the Hall current density, respectively, and $\boldsymbol{E}_{1}$ and $\boldsymbol{E}_{h}$ are their corresponding electric fields. $\rho, \mu$ and $\boldsymbol{R}_{H}$ are the electrical resistivity, the magnetic permeability and the Hall coefficient of materials, respectively. $\boldsymbol{R}_{H}$ of $\mathrm{Cu}$ and $\mathrm{CuNi}$ is set $-0.55 \times 10^{-10}$.

Resistivity of the cupper and SC-part (Rutherford cable) at the normal state are set as a function of magnetic flux density as in Table I. The side part of copper sheath shown in Fig. 1 is taken into account by assuming it as a member of the copper stabilizer of the Rutherford cable. Cross-sectional resistivity of the SC-part is considered as of a parallel circuit of the NbTi strands at normal state and the copper stabilizer.
TABLE I

REsistivity OF COPPER AND RUTHERFORD CABLE (SC) PART USED IN THE NUMERICAL SIMULATION

\begin{tabular}{lllll}
\hline \hline & $4 \mathrm{~T}$ & $5 \mathrm{~T}$ & $6 \mathrm{~T}$ & $7 \mathrm{~T}$ \\
\hline Copper & $2.93 \times 10^{-10}$ & $3.25 \times 10^{-10}$ & $3.75 \times 10^{-10}$ & $4.05 \times 10^{-10}$ \\
SC (Rutherford) & & & & \\
z-direction & $5.27 \times 10^{-10}$ & $5.82 \times 10^{-10}$ & $6.70 \times 10^{-10}$ & $7.22 \times 10^{-10}$ \\
y-direction & $1.05 \times 10^{-9}$ & $1.14 \times 10^{-9}$ & $1.27 \times 10^{-9}$ & $1.35 \times 10^{-9}$ \\
\hline \hline
\end{tabular}

The heat balance equation is given with temperature $T$ as

$$
\boldsymbol{C}(\boldsymbol{T}) \frac{\partial \boldsymbol{T}}{\partial \boldsymbol{t}}=\nabla \cdot(\boldsymbol{k}(\boldsymbol{T}) \nabla \boldsymbol{T})+\boldsymbol{Q}_{j}+\boldsymbol{Q}_{h},
$$

where $\boldsymbol{C}(\boldsymbol{T})$ and $\boldsymbol{k}(\boldsymbol{T})$ are the heat capacity and the thermal conductivity of materials, respectively. $\boldsymbol{Q}$ is the Joule heat density and $\boldsymbol{Q}_{h}$ is the heat input energy density (disturbance).

The finite differential method is applied to solve the above equations with the initial and boundary conditions. The mesh size $0.5 \mathrm{~mm}(\mathrm{z}) \times 0.22 \mathrm{~mm}(\mathrm{y})$ and the computation time step is $2.0 \mathrm{e}-7 \mathrm{~s}$. At the initial condition, all the transport current flows through the SC-part and the temperature of the whole conductor is equal to $\boldsymbol{T}_{b}$ of bulk Liq. He. At the both end of the conductor, the temperature is kept constant $\boldsymbol{T}_{b}$. At time $\boldsymbol{t}=0 \mathrm{~s}$, the pulsive heat input is applied for $0.1 \sim 1.0 \mathrm{~ms}$ at the top center part (10 $\mathrm{mm}$ length) of the conductor simulation model.

The heat transfer curves in Kapitza conductance regime (nonboiling regime) for bare and oxidized copper surface, respectively, are given by Iwamoto et al. [11]. These curves are expressed with the following equations.

$$
\begin{aligned}
& q=701.6\left(T_{C u}^{3.001}-T_{b}^{3.001}\right)(\text { Bare }) \\
& q=202.5\left(T_{C u}^{2.861}-T_{b}^{2.861}\right)(\text { Oxidized })
\end{aligned}
$$

The upper surface, that is, the cut off side is assumed as a bare (polished) copper. The lower side surface (Copper sheath) is oxidized one. $\boldsymbol{T}_{C u}$ is the surface temperature and $\boldsymbol{q}$ is the heat flux on the each surface. Heat transfer through the Kapton tape was taken into account.

In case of He II cooling, the heat flux from the cooling surface is limited by the Critical Heat Flux (CHF). The cooling surface of the conductor is assumed as a rectangular flat plate between the Kapton tapes. The rectangular cooling surface size changes with the length $l=0 \sim 37 \mathrm{~mm}$ according to the normal zone propagation with the width $w$ of $18 \mathrm{~mm}$ constant. Tatsumoto et al. [11] presented the correlation for the CHFs on a flat plate of width $w$ and length $l$ in a pool of pressurized He II. Based on the correlation, the average value of the CHF of the spreading rectangular cooling surface was used in the simulation.

The simulation was carried out with magnetic flux density from 4 to $7 \mathrm{~T}$ at the bulk He II temperature $2.0 \mathrm{~K}$.

\section{RESULTS AND DISCUSSION}

\section{A. Dynamic Normal Zone Propagation}

The simulation results were classified to three groups according to the transport current as was obtained by the experiments. First, only the normal zone initiated by the heat input re- 

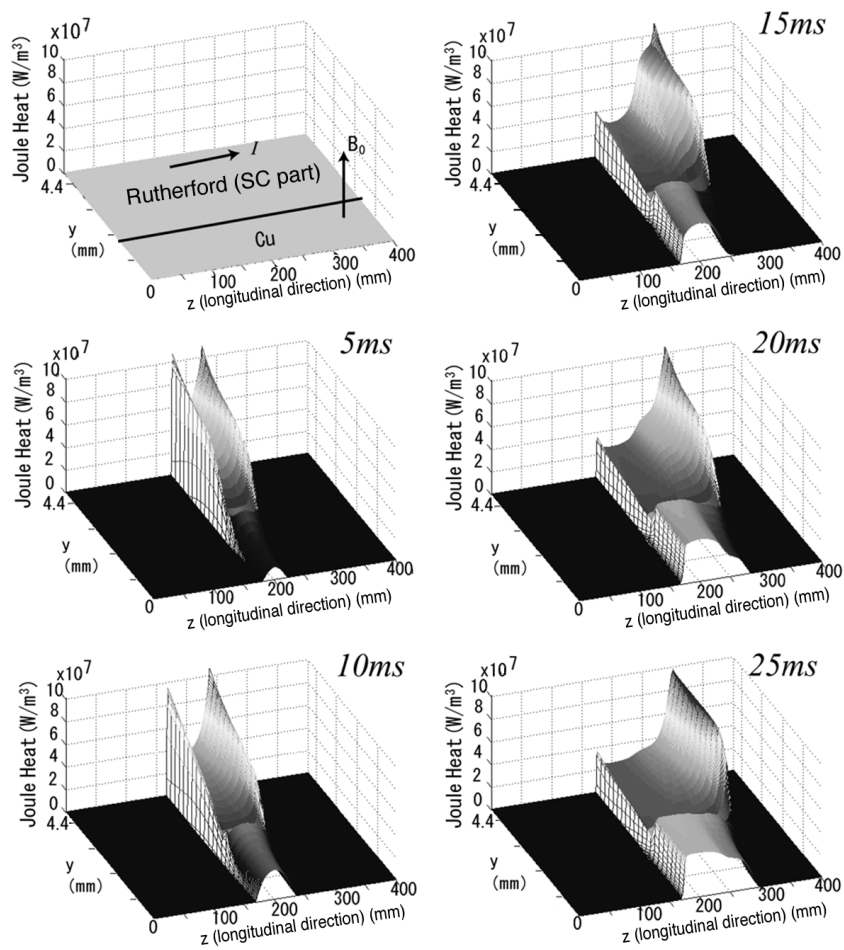

Fig. 3. Simulation result example of the Joule heat distribution along the conductor at the one-side propagation of normal zone. $\left(\boldsymbol{T}_{b}=2.0 \mathrm{~K}, \boldsymbol{B}_{0}=6 \mathrm{~T}\right.$, $I=15.3 \mathrm{kA})$.

mains around the heater but does not spread. Second, the normal zone spreads to one-side direction along the conductor. Third, the normal zone spreads both sides. The results showed asymmetrical propagations of the normal zone even in the conductor without the aluminum stabilizer.

Fig. 3 shows one of the simulation results of the Joule heat distribution which shows one-side propagation of the normal zone with $B_{0}=6 \mathrm{~T}$ and $I=15.3 \mathrm{kA}$.

The normal zone appeared due to the heat input at the longitudinal center of upper surface at $t=0 \mathrm{~ms}$. The transport current starts to shunt to the copper sheath and Joule heat is generated both in SC-part and the $\mathrm{Cu}$ Sheath. Even in the early stage $(t=5 \mathrm{~ms})$, asymmetrical Joule heat generation already appears. It is slightly larger in the SC-part near the Copper sheath at the right hand side. This unbalance of the heat generation in the SC part increases with time, and finally, the normal zone propagates only to the right hand direction.

However, the "traveling normal zone was not observed. The normal zone around the Kapton tape insulation parts once appeared does not disappeared.

Fig. 4 shows the simulation results of the LHD conductor with $\boldsymbol{B}_{0}=6 \mathrm{~T}$ and $\boldsymbol{I}=13.5 \mathrm{kA}$. Even at the time $10 \mathrm{~ms}$, the unbalance in the Joule heat distribution is larger than that for the Al-less test conductor. The large unbalance also can be seen in a thin layer of Al stabilizer part nearby the SC-part. It is confirmed that the Al stabilizer play a main role in the asymmetrical normal zone propagation. The normal zone once appeared soon disappeared and the normal zone of a finite size propagated, namely "traveling normal zone." It is expected that a $\mathrm{Cu}-2 \% \mathrm{Ni}$ layer with low thermal conductivity and an aluminum stabilizer with long current diffusion time cause the "traveling normal zone."

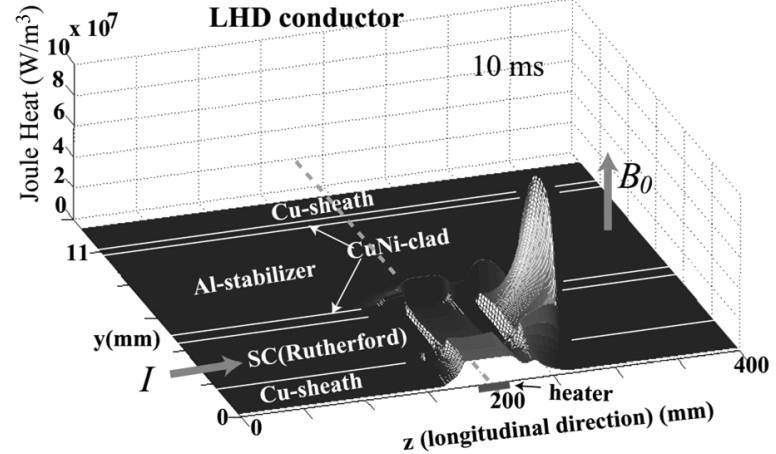

Fig. 4. Joule heat distribution along the conductor in LHD conductor $\left(T_{b}=\right.$ $\left.2.0 \mathrm{~K}, B_{0}=6 \mathrm{~T}, I=13.5 \mathrm{kA}\right)$.

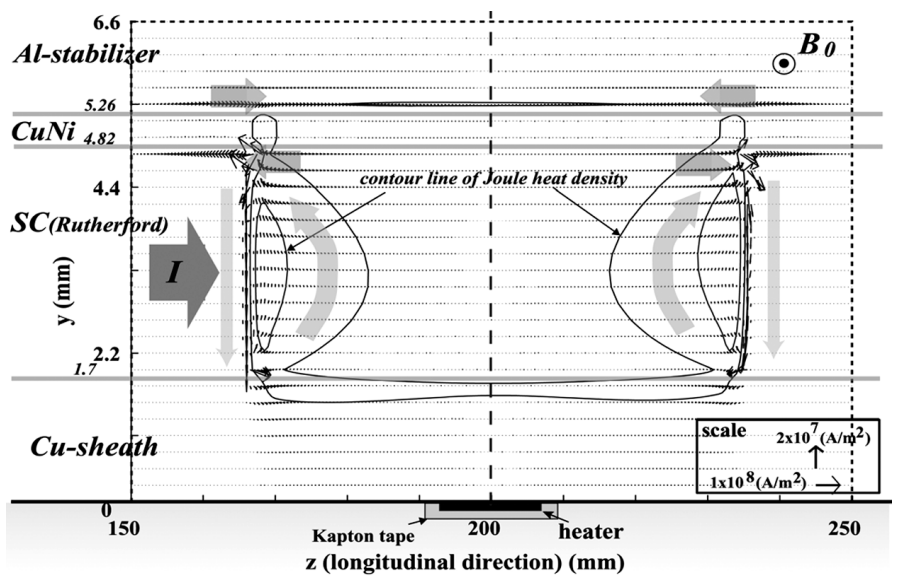

Fig. 5. Enlarged illustration of the Hall current flow near the center of the simulation model of LHD conductor $\left(t=4 \mathrm{~ms}, T_{b}=2.0 \mathrm{~K}, B_{0}=6 \mathrm{~T}\right.$, $I=13.5 \mathrm{kA})$.

\section{B. Hall Current Distribution}

Fig. 5 shows an enlarged illustration of the Hall current flow in the LHD conductor at $t=4.0 \mathrm{~ms}$ with conditions $\left(\boldsymbol{T}_{b}=\right.$ $\left.2.0 \mathrm{~K}, \boldsymbol{B}_{0}=6 \mathrm{~T}, \boldsymbol{I}=13.5 \mathrm{kA}\right)$. At the early stage of the current diffusion from $\mathrm{SC}$ part to stabilizers $(\mathrm{Al}, \mathrm{Cu})$ at the front of the normal zone, the simulation results showed the Hall current circulating between SC part and the aluminum stabilizer and also between SC part and copper sheath. The Hall coefficient $R_{H}$ of $\mathrm{Al}$ and $\mathrm{Cu}$ has different sign each other. The Hall current is added to the transport current and the current density unbalance in the SC-part of normal state, which has large resistivity, mainly causes the Joule heat unbalance. The Hall current in the SC-part has the same direction as the transport current around the right upper side of the normal zone front, then the lager Joule heat appears as shown in Fig. 4.

Fig. 6 shows the simulation results at $t=4.0 \mathrm{~ms}$ for the Al-less test conductor with conditions $\left(\boldsymbol{T}_{b}=2.0 \mathrm{~K}, \boldsymbol{B}_{0}=6 \mathrm{~T}\right.$, $I=15.3 \mathrm{kA}$ ). The Hall current circulating between SC part and copper sheath can be seen. The Joule heat unbalance occurs in the same way as in LHD conductor, but since the higher Joule heat density area is directly cooled by coolant (HeII), it is expected the unbalance of the temperature distribution along the conductor is smaller than that for LHD conductor.

The simulations with $\pm \boldsymbol{B}_{0}$ and $\pm \boldsymbol{I}$ were also performed. It is confirmed the one-side propagating direction depends on the 


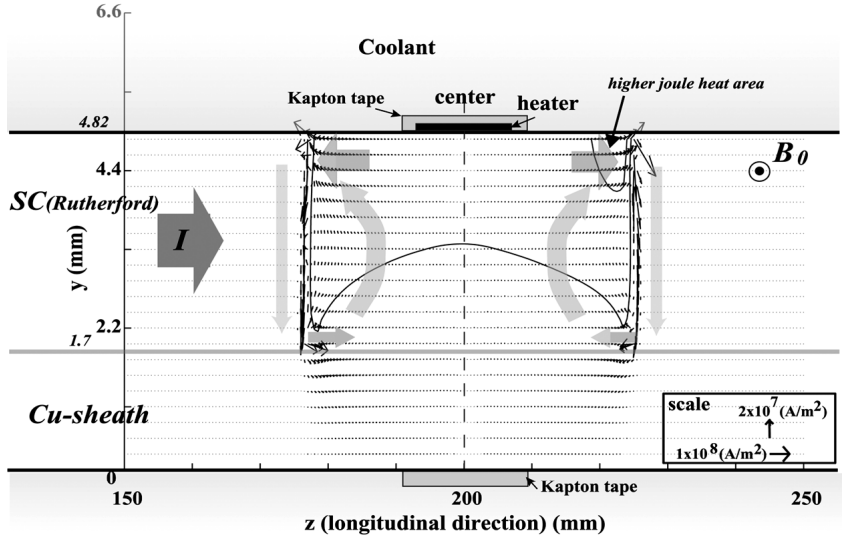

Fig. 6. Hall current flow near the center of the simulation model of Al-less test conductor $\left(t=4 \mathrm{~ms}, T_{b}=2.0 \mathrm{~K}, B_{0}=6 \mathrm{~T}, I=15.3 \mathrm{kA}\right)$.

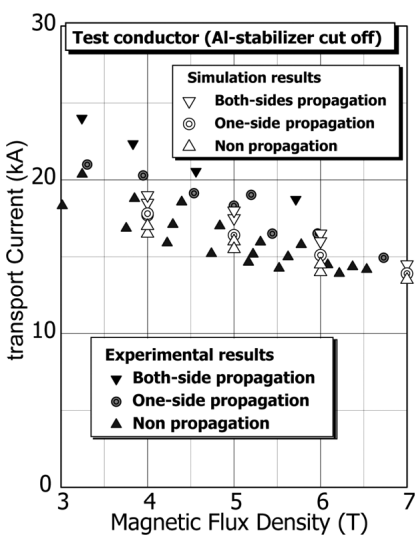

(a)

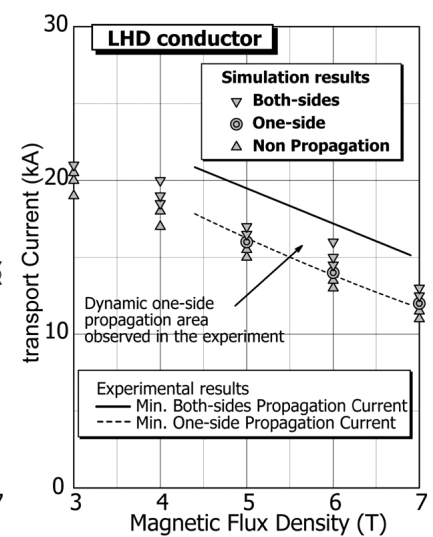

(b)
Fig. 7. Simulation result of stability limit with experimental results with shaded symbols: (a) Al-less test conductor; (b) LHD conductor.

direction of the magnetic field, but that of the transport current, since the one-side propagation is caused by the Hall current.

\section{Stability Simulation Results}

Fig. 7 shows the comparison of the stability simulation results with the experimental results for (a) the Al-less test conductor and (b) for the LHD conductor at $2.0 \mathrm{~K}$ of helium temperature. The current range for the one-side propagation is seen for both conductors, but that for test conductor is narrower than for LHD one. However, the experimental results have much wider current range for one-side propagation for both conductors.

It is expected that the 3-dimensional Hall current flow contributes the stronger asymmetrical normal zone propagation in LHD conductor due to $\mathrm{Cu}$-sheath and mainly $\mathrm{Al}$ stabilizer with asymmetrical configuration.

\section{CONCLUSION}

In order to study the effects of the aluminum stabilizer of LHD conductor on its transient stability, the simulation studies for $\mathrm{Al}$ cut off LHD conductor were carried out. The results obtained are as follows.

Asymmetrical propagation of the normal zone appears even in the LHD based conductor without the aluminum stabilizer.
The simulation results show that the asymmetrical configuration of the superconductor strands and stabilizer materials causes the asymmetrical propagation phenomenon.

The range of the transport current for the one-side propagation of the normal zone, is narrower than that for the LHD conductor. It is confirmed that the aluminum stabilizer plays main role in the asymmetrical normal zone propagation.

In the case of the He II cooling, at the same external magnetic flux density, the transport current that initiates the traveling normal zone for the LHD conductor is almost equal to that for the Al-less test conductor. The aluminum stabilizer has little contribution to the transient stability of the conductor because of its long current diffusion time.

Because of the low thermal conductivity of CuNi clad, it is considered that the stability of the LHD conductor was mainly determined by the cooling features from the lower copper sheath surface. On the contrary, the Al-less test conductor has very small margin in steady state stability, however, since the Rutherford cable is directly cooled by Liq. He II, the high performance of the He II cooling in the transient state makes the normal zone initiating current up to the same level of that for the super-stabilized LHD conductor.

These results and the proposed simulation code will contribute to the design of future large scale superconductors immersed in sub-cooled He I or He II.

\section{REFERENCES}

[1] N. Yanagi, S. Imagawa, T. Mito, A. Iwamoto, H. Chikaraishi, S. Hamaguchi, A. Nishimura, T. Satow, Y. Nakamura, and S. Satoh, "Analysis of the normal transition event of the LHD helical coils," IEEE Trans. Appl. Supercond., vol. 10, pp. 610-613, 2000.

[2] A. Higuchi, M. Ohya, Y. Shirai, M. Shiotsu, and S. Imagawa, "Cooling stability test of He II cooled LHD conductor (1) - Current supply and measuring method-," IEEE Trans. Appl. Supercond., vol. 14, pp. 1443-1446, 2004.

[3] M. Ohya, A. Higuchi, Y. Shirai, M. Shiotsu, and S. Imagawa, "Cooling stability test of He II cooled LHD conductor (2)—Experimental results_," IEEE Trans. Appl. Supercond., vol. 14, pp. 1447-1450, June 2004.

[4] A. Devred, "Investigation on the normal zone along a layer of thermally insulated superconductors," J of Appl. Phys., vol. 67, pp. 7467-7477, 1990.

[5] R. Kupferman, R. G. Mints, and E. Ben-Jacob, "Propagating normal domains in large composite superconductors," J of Appl. Phys., vol. 70, pp. 7484-7491, 1991.

[6] A. Lee, R. H. Wands, and R. W. Fast, "Study of current redistribution in an aluminum stabilized superconductor," Cryogenics, vol. 32, pp. 863-866, 1992.

[7] N. Yanagi, S. Imagawa, Y. Hishinuma, K. Seo, K. Takahata, S. Hamaguchi, A. Iwamoto, H. Chikaraishi, H. Tamura, S. Moriuchi, S. Yamada, A. Nishimura, T. Mito, and O. Motojima, "Asymmetrical normal-zone propagation observed in the aluminum-stabilized superconductor for the LHD gelical coils," IEEE Trans. Appl. Supercond., vol. 14, pp. 1517-1510, 2004.

[8] T. Tsuchiya, S. Noguchi, and H. Yamashita, "Transient stability analysis of large aluminum stabilized superconductor by $2 \mathrm{D}$ and $3 \mathrm{D}$ finite element analysis," IEEE Trans. Appl. Supercond., vol. 14, pp. 1330-1333, 2004.

[9] M. Ohya, Y. Shirai, M. Shiotsu, and S. Imagawa, "Numerical analysis on dynamic one-side propagation of normal zone observed in LHD conductor," in Advances in Cryogenic Engineering, Trans. Cryogenic Engineering Conference-CEC, 2006, vol. 51.

[10] R. Ikuta, M. Ohya, Y. Shirai, M. Shiotsu, and S. Imagawa, "Effect of aluminum stabilizer on transient stability of LHD conductor cooled by pressurized He II (1)-Experimental results—," presented at the ASC2006, , unpublished.

[11] A. Iwamoto, R. Maekawa, and T. Mito, "Kapitza conductance of an oxidized copper surface in saturated He II," Cryogenics, vol. 41, pp. $367-371,2001$.

[12] H. Tatsumoto, K. Hata, K. Takeuchi, Y. Hama, Y. Shirai, and M. Shiotsu, "Critical heat flux on various sized flat plates in pressurized He II," in ICEC 17, 1998, pp. 683-690. 